\title{
Systeme zur Assistenz und Effizienzsteigerung in manuellen Produktionsprozessen der Industrie auf Basis von Projektion und Tiefendatenerkennung
}

\author{
Andreas Bächler, Liane Bächler, Sven Autenrieth, Hauke Behrendt, \\ Markus Funk, Georg Krüll, Thomas Hörz, Thomas Heidenreich, Catrin \\ Misselhorn und Albrecht Schmidt
}

\subsection{Motivation}

Der demografische Wandel und die Globalisierung führen zu erheblichen Veränderungen der heutigen Industrieprozesse der Produktion und der dazugehörigen Logistik in Deutschland. Eine Folge der Globalisierung ist die Entwicklung des Marktes vom Anbieter- über den Käufer- hin zum Individualmarkt (Günthner et al. 2004; Lotter 2012). Dazu gehört die Möglichkeit der individuellen Gestaltung von Produkten, aber vor allem auch die ständige

\footnotetext{
A. Bächler $(\square)$

RITTAL GmbH \& Co. KG, Rudolf Loh Straße 2, D-35708, Haiger, Deutschland e-mail: baechler.a@ rittal.de

L. Bächler $\cdot$ T. Heidenreich

Social Work, Health Care and Nursing Sciences,

University of Applied ScienceEsslingen, Deutschland

e-mail: Liane.baechler@hs-esslingen.de

S. Autenrieth · G. Krüll · T. Hörz

Mechanical Engineering, University of Applied ScienceEsslingen, Esslingen am Neckar, Deutschland

H. Behrendt · C. Misselhorn

Institut für Philosophie, Universität Stuttgart, Stuttgart, Deutschland

M. Funk

Institut for Visualization and Interactive Systems, University of Stuttgart,

Stuttgart, Deutschland
A. Schmidt
University of Stuttgart, Stuttgart, Deutschland. 
Verfügbarkeit von Waren und Informationen (Ten Hompel et al. 2011). Der Trend der kundenindividuellen Produkte mit der Losgröße 1 verursacht eine hohe Variantenvielfalt und eine steigende Komplexität im Produktions- und Logistiknetzwerk (Reif 2009). Diese Veränderung von der Großserienfertigung hin zu einer Fertigung mit geringen Stückzahlen führt dazu, dass sich die Anzahl repetitiv auszuführender Tätigkeiten reduziert und sich somit automatisierte Lösungen in der Fertigung oftmals nicht mehr wirtschaftlich einsetzen lassen. Eine fehlende Flexibilität, hohe Investitions- bzw. Betriebskosten, aber auch der Bedarf an höher qualifizierten Mitarbeitern für die Bedienung und Wartung dieser vollautomatisierten Anlagen erschweren zusätzlich den Einsatz in Produktions- und Logistikprozessen (Lotter 2012).

Um in diesen Veränderungen auf einem globalen Markt wettbewerbsfähig bleiben zu können, sind sowohl eine hohe Flexibilität der Produktion mit kurzen Montage-, Kommissionier-, Rüst- und Einlernzeiten als auch ein hoher Qualitätsstandard und niedrige Produktions- und Logistikkosten erforderlich.

Der Mensch bietet mit seiner Eigenschaft der komplexen Wahrnehmung, seinem Greif-, Tast-, Hör- und Sehvermögen sowie durch seine kognitiven Fähigkeiten geeignete Voraussetzungen, um schnell und flexibel auf die sich verändernden Markt- und Produktionsbedingungen zu reagieren (Arnold und Furmans 2009; Böhle 2005).

Die durch die beschriebenen Entwicklungen verursachten häufig auftretenden Änderungen in den manuellen Arbeitsabläufen führen einerseits zu einem abwechslungsreicheren und dadurch interessanteren Arbeitsinhalt der Mitarbeiter. Andererseits steigen aber auch die Ansprüche hinsichtlich ihrer Leistungsfähigkeit, Ausdauer und Konzentration und dadurch auch der Fehleranfälligkeit, je länger die Mitarbeiter mit ihrer Tätigkeit befasst sind (Reinhart und Zäh 2014). Aufgrund der hohen Varianz der Produkte ist zur Sicherstellung einer gleichbleibend hohen Qualität der manuellen Tätigkeiten eine ebenfalls hohe Qualifikation der Mitarbeiter erforderlich.

Neben den industrieseitigen Veränderungen stellt der demografische Wandel mit einer stetig älter werdenden Gesellschaft eine zusätzliche Herausforderung dar. Aktuelle Zahlen des Statistischen Bundesamtes zeigen, dass sich das Durchschnittsalter der Erwerbstätigen und dadurch der Anteil der Beschäftigten, die ein eingeschränktes Leistungsspektrum aufweisen können, in Deutschland deutlich erhöhen. Konkret wird von 2000-2050 ein Rückgang des Bevölkerungsstandes in Deutschland um $9 \%$ prognostiziert. Der Anteil der für die Industrie wichtigen Gruppe der 20- bis 60-Jährigen sinkt dabei von 45,5 auf 35,4 Mio. Erwerbstätige. Die Zahl der über 60-Jährigen steigt hingegen von 19,4 auf 27,5 Mio. Menschen an (Statistisches Bundesamt 2009). Diese Zahlenprognosen erfolgten jedoch ohne Berücksichtigung der aktuell starken Zuwanderung durch Migranten und Flüchtlinge. Das Ergebnis einer Marktstudie mit 130 in Deutschland ansässigen 
Industrieunternehmen zeigt, dass der Anteil an leistungsgewandelten ${ }^{1}$ Beschäftigten bereits heute einen prägnanten Wert von bis zu $20 \%$ der Gesamtbelegschaft einnimmt (Hörz et al. 2013).

Die „Engpassanalyse 2013“ des Bundesministeriums für Wirtschaft und Energie beschreibt den ebenso durch die überalternde Gesellschaft bedingten Fachkräftemangel in den industriellen Berufsfeldern. Dieser Mangel an Nachwuchs- und Fachpersonal führt dazu, dass Unternehmen immer häufiger ihre Bedarfe in den jeweiligen Tätigkeitsfeldern nicht adäquat decken können (Bundesministerium für Wirtschaft und Technologie (BMWi) 2013). Zusätzlich führen die steigenden Anforderungen an Werkstätten für behinderte Menschen (WfbM) dazu, dass für die Personengruppe der leistungsgeminderten Menschen neue Wege der Beschäftigung und dadurch auch der Unterstützung gefunden werden müssen.

Aufgrund dessen ist es nicht nur aus sozialen, sondern auch aus wirtschaftlichen Aspekten erforderlich, leistungsgewandelte und leistungsgeminderte ${ }^{2}$ Menschen im industriellen Umfeld weiter bzw. neu zu beschäftigen (Bundesministerium für Arbeit und Soziales 2014). Von Seiten der Industrie haben die Bemühungen für eine inklusive Gestaltung des Arbeitsmarktes ebenfalls zugenommen, so wie dies im Übereinkommen über die Rechte von Menschen mit Beeinträchtigungen (UN-Behindertenrechtskonvention) gefordert wird und im Sozialgesetzbuch IX mit dem Gesetz zur „Rehabilitation und Teilhabe behinderter Menschen“" geregelt ist (Beauftragte der Bundesregierung für die Belange behinderter Menschen 2014; Bundesministerium für Arbeit und Soziales 2011; Artikel des Sozialgesetzbuches - Neuntes Buch - (SGBIX) Rehabilitation und Teilhabe behinderter Menschen 2001).

\subsection{Forschungsprojekt motionEAP}

Im Zuge des Forschungsprojektes „motionEAP - System zur Effizienzsteigerung und Assistenz bei Produktionsprozessen in Unternehmen auf Basis von Bewegungserkennung und Projektion“"werden anwender- und prozessorientierte Assistenzsysteme für Montageund Kommissionierprozesse entwickelt, prototypisch umgesetzt und evaluiert. Der nutzerorientierte Entwicklungsprozess soll dabei sowohl die Anforderungen der Industrieunternehmen als auch die der leistungsgeminderten Anwender miteinander kombinieren. Die

\footnotetext{
${ }^{1}$ Unter „leistungsgewandelten“ Mitarbeitern sind ehemals vollleistende Beschäftigte mit Einsatzeinschränkungen zu verstehen, welche für einen gewissen Zeitraum oder dauerhaft nicht mehr in der Lage sind, ihre bisherige Arbeitstätigkeiten mit den entsprechenden Anforderungen und Belastungen auszuführen. Hierbei geht es besonders um Mitarbeiter mit erworbenen Behinderungen als Folge einer Krankheit, eines Unfalls oder von Alterserscheinungen. An einem angepassten Arbeitsplatz können diese Mitarbeiter jedoch ihre volle Leistung erbringen (Adenauer 2004; Jahn 2001).

${ }^{2}$ Im vorliegenden Artikel werden Personen mit Beeinträchtigung der funktionalen Gesundheit als „leistungsgeminderte“ Mitarbeiter bezeichnet. Dieser Begriff bezieht sich auf eine Einschränkung der Leistungsfähigkeit, womit das maximale Leistungsniveau einer Person bzgl. einer Aufgabe oder Handlung unter Test-, Standard oder hypothetischen Bedingungen gemeint ist (Schuntermann 2007).
} 
Entwicklung und Umsetzung erfolgt in einer interdisziplinären Zusammenarbeit mit Experten aus den Fachgebieten Maschinenbau, Informatik, Pädagogik, Psychologie und Ethik.

\subsubsection{Ziele und Herausforderungen}

Mit den Assistenzsystemen werden folgende Ziele verfolgt (siehe Abb. 3.1):

- Die Komplexität und der Aufwand bei der Einarbeitung von Mitarbeitern mit unterschiedlichem Leistungsniveau und fachlichem Hintergrund in neue Tätigkeitsgebiete soll reduziert werden.

- Die Motivation und Arbeitsfähigkeit von älteren und leistungsgeminderten Mitarbeitern soll erhalten bzw. verbessert und die Anzahl an Produktionsfehlern reduziert werden.

- Die kognitive Beanspruchung und das Stresslevel der Mitarbeiter während der Arbeitstätigkeit soll verringert werden.

- Die Einbeziehung ergonomischer und motivierender Elemente soll verschleißbedingten Erkrankungen vorbeugen und ein gesundes Arbeitsverhalten unterstützen.

- Die Inklusion und Wiedereingliederung von leistungsgeminderten und -gewandelten Personen in industrielle Arbeitsumgebungen soll aufgrund sozialer und wirtschaftlicher Gesichtspunkte ermöglicht werden.

- Die Wettbewerbsfähigkeit von deutschen Unternehmen und WfbMs bei manuellen Industrietätigkeiten soll verbessert werden.

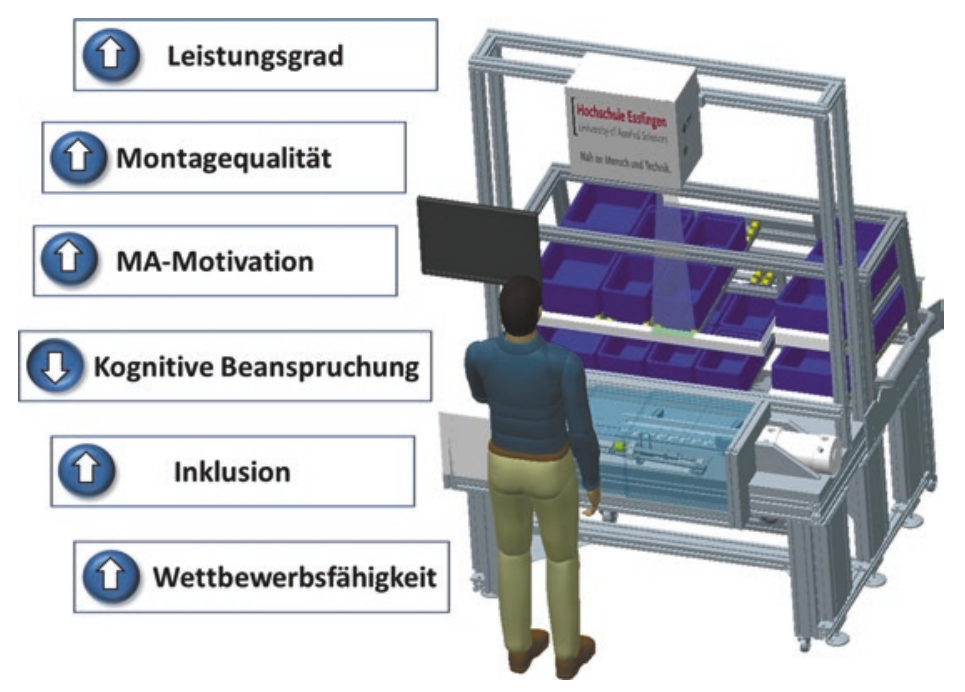

Abb. 3.1 Ziele des Assistenzsystems 


\subsubsection{Technische Umsetzung}

Im Zuge des Projektes wird ein Assistenzsystem für Montage- und eines für Kommissionierprozesse entwickelt (siehe Abb. 3.2). Das Assistenzsystem für Montageprozesse wird dabei in zwei Anwendungsszenarien eingesetzt. Zum einen findet das Assistenzsystem Anwendung in einer Montagezelle mit drei verketteten Arbeitsplätzen zur Unterstützung der Montage von PKW-Anlassern (siehe Abb. 3.2). Zum anderen wird das System an einem Einzelarbeitsplatz zur Montage von Schraubzwingen eingesetzt (siehe Abb. 3.1 oder Abb. 3.3).

\subsubsection{Anwendungsfall Montage}

Das motionEAP-Montageassistenzsystem besteht aus zwei Hauptkomponenten: einem Kamerasystem (Microsoft Kinect) und einem Projektierungsgerät (Beamer), welche über einen PC mit einer speziell dafür entwickelten Assistenzsoftware gesteuert werden (siehe
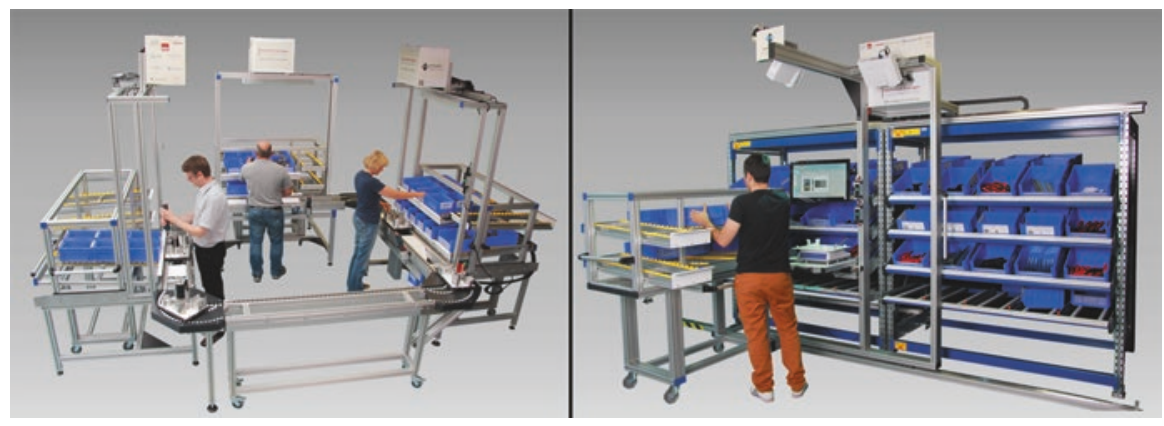

Abb. 3.2 Assistenzsysteme in einer Montagezelle (links), Assistenzsystem für Kommissionierprozesse (rechts)

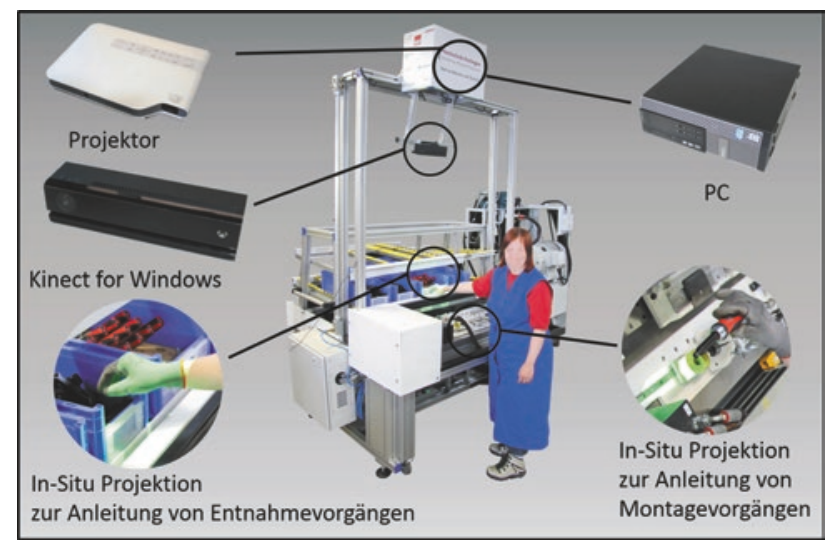

Abb. 3.3 Hauptbestandteile und Anleitungsfunktionen des Montageassistenzsystems 
Abb. 3.3). Diese Komponenten können durch ihren modularen Hardwareaufbau an bzw. über fast jedem herkömmlichen Montagearbeitsplatz angebracht werden (vgl. Funk et al. 2015b).

Das System benutzt die Tiefendaten der Microsoft Kinect_v2, um Arbeitsprozesse der Mitarbeiter am Arbeitsplatz zu erkennen. Dabei erfolgt eine Unterscheidung zwischen Entnahme- und Montagevorgängen. Bei der Entnahme wird über jedem Kleinladungsträger (KLT), in dem Teile für den Montageprozess aufbewahrt werden, eine interaktive Zone erstellt. Diese interaktive Zone umfasst den Bereich über dem KLT mit einer Höhe von ca. $15 \mathrm{~cm}$. Wenn ein Mitarbeiter ein Bauteil aus einem KLT entnimmt, führt dies zu einer Veränderung der Tiefendaten in diesem Bereich und das Assistenzsystem erkennt einen Entnahmevorgang. Die zweite Aktion ist das automatische Erkennen der korrekten Montage der Teile. Dabei überwacht die Kinect die aktuellen Tiefendaten des Montagebereichs mit 30 Bildaufnahmen pro Sekunde und vergleicht diese mit einem zuvor referenzierten Soll-Zustand. Um Sensorrauschen und Toleranzen auszugleichen, gilt ein Teil als korrekt verbaut, wenn mindestens $85 \%$ der Tiefendaten mit dem Referenzwert übereinstimmen. Dieser Wert wurde empirisch ermittelt und hat sich in mehreren Studien als zuverlässig erwiesen. Nachdem die Montage eines Bauteils korrekt erkannt wurde, wird der nachfolgende Arbeitsschritt angeleitet.

Zur Unterstützung von unerfahrenen oder leistungsgeminderten Mitarbeitern bei deren Arbeitstätigkeiten wird durch das Assistenzsystem ein Feedback ,in-situ“ projiziert, d. h. Informationen direkt auf dem Arbeitsplatz bereitgestellt. Dabei wird in einem ersten Schritt die Entnahme und die Anzahl der Bauteile aus dem entsprechenden KLT über einen grün blinkenden Lichtbalken und die Entnahmestückzahl, welche vor den KLT projiziert werden, angeleitet (siehe Abb. 3.3). Falls ein Mitarbeiter versucht, ein Teil aus einem falschen KLT zu entnehmen, wird dies mit einem roten Balken hervorgehoben. Diese Art der Fehlerrückmeldung soll die Mitarbeiter auf entsprechende Fehler aufmerksam machen. Nach der korrekten Entnahme der Bauteile wird die korrekte Montageposition und Bauteilorientierung durch die Projektion der grün leuchtenden Kontur des Bauteils an der entsprechenden Position auf der Montagevorrichtung angeleitet (siehe Abb. 3.3). In vergangenen Studien hat sich diese Anleitungsform mittels grüner Kontur neben einer mündlichen Einweisung, einer Bildanleitung und einer Videoanleitung als die hilfreichste Darstellungsform in Bezug auf die subjektive Beanspruchung, die Ausführungszeit und die Fehlerrate erwiesen (Funk et al. 2015a). Nach der korrekten Positionierung und Montage eines Bauteils startet das Assistenzsystem mit der Entnahmeanleitung des nachfolgenden Bauteils.

Durch die Erfassung und Erkennung von Arbeitsabläufen, Zeiten und Fehlern ist das System in der Lage, den Lernzustand bzw. den Erfahrungsstand des Mitarbeiters einschätzen zu können. Hierdurch kann das System automatisch auf den Mitarbeiter ,zugeschnittene“ Anleitungen mit niedrigerem oder höherem Informationsgehalt bzw. mehr oder weniger visuelles Feedback bieten. Beim vorliegenden Assistenzsystem werden folgende drei Feedback-Level definiert: Anfänger, Fortgeschrittener und Experte. Im Anfänger-Level wird zusätzlich zu der Entnahme- und Montageposition jedes Bauteils eine Videoanleitung angezeigt, die dem Mitarbeiter die Montageschritte und -inhalte nochmals anzeigt. 
Die dauerhafte Bereitstellung von Videoanleitungen wirkt sich störend aus, deshalb erfolgt die Anleitung mit steigender Arbeitserfahrung im Fortgeschrittenen-Level mittels projizierter Konturen. Im Expertenmodus bietet das System keinerlei visuelle Anleitungen für die Montageschritte, jedoch erkennt und kontrolliert das System in diesem Level die Montage- und Entnahmeschritte im Hintergrund und kann dadurch im Fall eines Fehlers intervenieren.

Das Einrichten eines neuen Montageprozesses und die Erstellung der interaktiven Anleitungen werden durch eine Funktion zur Programmierung durch Demonstration vereinfacht. Hierbei ,programmiert“ ein Einrichter, welcher den Montageprozess bereits kennt, den Prozess durch einmaliges „Vormachen“ der Arbeitsschritte und -abfolge. Das Assistenzsystem erkennt im Einrichtemodus während dem Vormachen die einzelnen Arbeitsschritte und speichert für jedes Bauteil einen Entnahme- und Montageschritt ab. Dabei werden für die Entnahmeschritte der Entnahmeort und für die Montageschritte nach dem korrekten Verbau des jeweiligen Bauteils die Tiefendaten gespeichert. Mithilfe dieser Daten errechnet das Assistenzsystem bei den anschließenden Montagetätigkeiten automatisch die Position und Orientierung der projizierten Lichtbalken und Konturen. Zusätzlich dienen die Daten als Referenzwerte bzw. Soll-Zustände für die Kontrolle der korrekten Entnahme- und Montageposition bzw. Orientierung der einzelnen Bauteile. Durch die Funktionsweise dieses Einrichtemodus ist kein kompliziertes und zeitintensives Programmieren oder Einrichten von neuen Montageprozessen erforderlich. Nach abgeschlossenem Einrichtevorgang werden mithilfe der Anleitung die Mitarbeiter mit unterschiedlichen Leistungsniveaus dazu befähigt, ihnen noch unbekannte Montagetätigkeiten bereits beim ersten Durchlauf selbstständig durchzuführen.

\subsubsection{Pädagogisch-Psychologische Aspekte für die Nutzung von Assistenzsystemen}

In den letzten Jahren hat sich international ein verändertes Paradigma in Bezug auf die Inklusion von Menschen mit Behinderungen durchgesetzt. Niedergeschlagen hat sich dies insbesondere in der UN-Behindertenrechtskonvention über die Teilhabe von Menschen mit Behinderung und beeinflusst indessen die Diskussion auf gesamtpolitischer Ebene. Durch die Ratifizierung dieser Konvention im Jahr 2009 wurde Inklusion somit zum Leitgedanken und zum zentralen Handlungsprinzip, auch innerhalb der Bundesrepublik. ${ }^{3}$ So

\footnotetext{
${ }^{3}$ Zwar waren in der deutschen Gesetzgebung schon vorher einige Regelungen enthalten, um die Gleichberechtigung von Menschen mit Behinderung durchzusetzen, wie z. B. das Grundgesetz zur Verhinderung der Benachteiligung von Menschen mit Behinderung (Art. 3 Abs. 3). Auch das Behindertengleichstellungsgesetz (BGG) verfolgt dieses Ziel, zudem ist das Recht auf Teilhabe am gesellschaftlichen Leben im Sozialgesetzbuch festgelegt (SGB IX). Dennoch gibt es viele Bereiche, in denen die UN-Konvention detaillierte Hinweise und somit der deutschen Gesetzgebung wichtige Impulse zur Gestaltung der Inklusion von Menschen mit Behinderungen gibt.
} 
besagt Art. 4 der Behindertenrechtskonvention, dass unter anderem Geräte und neue unterstützende Technologien einen entscheidenden Beitrag zur Ausgestaltung einer inklusiven Gesellschaft, insbesondere hinsichtlich des Arbeitsmarktes, darstellen. In Bezug auf den Arbeitsplatz bedeutet dies, dass angemessene Vorkehrungen für Menschen mit Behinderungen getroffen werden müssen, welche ihre Einschränkungen und Bedürfnisse berücksichtigen (vgl. Art. 27) (Bundesministerium für Arbeit und Soziales 2011).

Forschungstätigkeiten im Themengebiet der Erwerbslosigkeit zeigen, dass insbesondere Erwerbsarbeit eine wichtige sinn- und identitätsstiftende Funktion im Leben darstellt (Schaper 2006). In unserem westlichen Kulturkreis erfüllt sie nicht nur Funktionen der Existenzsicherung, sondern stellt gleichzeitig den wichtigsten Ursprung für die Gestaltung der persönlichen Identität und für vielfältige Lebenserfahrungen dar. Die zahlreichen Folgewirkungen von Erwerbslosigkeit, wie z. B. Langeweile, emotionale Labilität, Gefühle der Wertlosigkeit und des Überflüssigseins oder psychosomatische Beschwerden, verdeutlichen diese Umstände und zeigen das Ausmaß der Notwendigkeit einer Teilhabe an Arbeit (Schwab 2009; Semmer und Udris 2004).

Die vorgestellten Assistenzsysteme berücksichtigen diese aktuellen Forderungen und unterstützen somit die Inklusion und Partizipation an der Arbeitswelt vor allem von Menschen mit Einschränkungen. Dabei geht es um eine für die Anwendungsgruppe zielgerichtete Unterstützung, welche diese nicht über-, aber gleichfalls auch nicht unterfordert.

\subsubsection{Ethische Implikationen von Assistenzsystemen am Arbeitsplatz}

Technologische Innovationen haben das Potenzial, die Lebenswelt des Menschen fundamental zu verändern. Die ethische Untersuchung von Assistenzsystemen am Arbeitsplatz beinhaltet die Antizipation und ethische Bewertung solcher Auswirkungen, die diese Technologien auf die Arbeitswelt und unser Verständnis von Arbeit haben können. Wichtige Gesichtspunkte der ethischen Bewertung von Assistenzsystemen am Arbeitsplatz sind beispielsweise die Bedeutung der Arbeit für das gute Leben, die Merkmale guter oder zumindest menschenwürdiger Arbeit und Arbeitsbedingungen sowie die gerechte Verteilung von Arbeit.

Die Untersuchung dieser Gesichtspunkte lässt sich in negative und positive Aspekte unterteilen. Negative Aspekte geben Gründe, die gegen einen Einsatz von Assistenzsystemen am Arbeitsplatz sprechen; positive Aspekte entsprechend Gründe für einen solchen. Diese Aspekte müssen gegeneinander abgewogen werden. Im Fall von Konflikten muss erwogen werden, wie die ethisch positiven Aspekte unter Vermeidung möglicher negativer Konsequenzen realisierbar sind. Auf eine griffige Formel gebracht geht es bei der Untersuchung ethischer Implikationen von Assistenzsystemen am Arbeitsplatz also um die Frage, welchen Wert diese Technologie für die gesellschaftliche Entwicklung besitzt und worin dieser Wert im Einzelnen besteht. 
Die ethische Beurteilung konkreter Sachverhalte erfordert eine Herangehensweise, die den gesellschaftlichen Kontext miteinbezieht. Für diesen speziellen Fall muss etwa berücksichtigt werden, dass es um den Einsatz von Assistenzsystemen am Arbeitsplatz und nicht in der Privatsphäre geht, dass dieser Arbeitsplatz in einer kapitalistisch organisierten Arbeitswelt verortet ist und dass diese wiederum als Teil eines umfassenden gesellschaftlichen Kooperationszusammenhangs gesehen werden muss. Um dabei eine möglichst präzise Einschätzung der ethischen Dimension von Assistenzsystemen am Arbeitsplatz abgeben zu können, ist es hilfreich, zwei Fragen zu unterscheiden:

- Wie sehen Standards guter Arbeit aus, die festlegen, unter welchen Bedingungen Assistenzsysteme eingesetzt werden müssen?

- Warum sollten Assistenzsysteme überhaupt eingesetzt werden?

Die erste Frage thematisiert, wie Assistenzsysteme eingesetzt werden müssen, um ethischen Grundsätzen zu entsprechen. Die allgemeinen ethischen Standards, an denen heute ein guter Arbeitsplatz gemessen wird, dürften sich ihrer Struktur nach auf den speziellen Fall von Arbeitsplätzen mit Assistenzsystemen übertragen lassen. Ein allgemeines moralisches Prinzip, dem diese Standards entsprechen müssen, lässt sich in Anlehnung an Kant als „Instrumentalisierungsverbot“ bezeichnen (Kant 1961). An die Stelle von gesundheitlichen Belastungen, restriktiven Arbeitsbedingungen, monotonen Arbeitsinhalten und autoritärer Führung setzen diese Standards Vorstellungen von guter Arbeit, in denen ein Bedürfnis nach Selbstbestimmung, Anerkennung und Beteiligung ausschlaggebend ist (Sauer 2011). Es ist daher davon auszugehen, dass sich Arbeiter, die mit einem Assistenzsystem arbeiten, als weitestgehend selbstbestimmt und eigenverantwortlich verstehen können müssen. Andernfalls wäre die wichtige Forderung nach Autonomie verletzt. Gleichzeitig dürfen die besonderen Bedürfnisse von Menschen mit Behinderung nicht unberücksichtigt bleiben. Assistenzsysteme können gerade für diese Personengruppe hilfreich sein, wenn und sofern auf einen bedarfsgerechten Einsatz geachtet wird. Neben dem Instrumentalisierungsverbot gilt für Arbeitsplätze mit Assistenzsystem also auch eine gewisse Fürsorgepflicht. Um diese unscharfen Begriffe handhabbar zu machen, müssen die Perspektiven von allen beteiligten Personen genauer betrachtet und ihre begründeten Ansprüche gegeneinander abgewogen werden.

Eine umfassende Untersuchung ethischer Aspekte kann sich jedoch nicht mit dem Nachweis zufrieden geben, dass der Einsatz von Assistenzsystemen am Arbeitsplatz unter bestimmten Bedingungen moralisch erlaubt ist. Sie muss sich ebenfalls mit der zweiten Frage beschäftigen, warum Assistenzsysteme eingesetzt werden sollten, welche positiven Gründe also für einen Einsatz sprechen. Ein wichtiges Einsatzgebiet betrifft die Inklusion leistungsgeminderter und -gewandelter Menschen (vgl. Abschn. 3.2.3). Assistenzsysteme öffnen dieser Personengruppe einen Teil der Arbeitswelt, der ihnen ohne sie verschlossen bliebe oder von dem sie zumindest eklatant von Ausschluss bedroht wären. Unter der Voraussetzung, dass Arbeit in kapitalistischen Gesellschaften 
eine wichtige Dimension des guten Lebens und der gesellschaftlichen Teilhabe darstellt, spricht die Inklusion leistungsgeminderter Menschen auf den ersten Blick ethisch für den Einsatz von Assistenzsystemen. Hierbei sind auch die ethischen Auswirkungen zu prüfen, die der Einsatz von Assistenzsystemen auf die gerechte Verteilung von Arbeit haben könnte.

Die positive Bewertung der Arbeit muss freilich selbst einer genauen ethischen Überprüfung unterzogen werden. Lässt sie sich mit Blick auf das aufgeklärte Eigeninteresse der Beteiligten als gewollt ausweisen, spiegelt sie einen allseitig geteilten Wert wider bzw. hilft dabei, diesen gesellschaftlich zu verwirklichen, oder handelt es sich gar um eine stärkere moralische Forderung? Antworten auf diese Frage verbessern unser Verständnis der Gründe, die für den Einsatz von Assistenzsystemen am Arbeitsplatz sprechen. Dies ist wichtig, um eine unter ethischen Gesichtspunkten positive Ausgestaltung des Einsatzgebiets dieser Systeme zu realisieren.

\subsection{Auswirkungen von Assistenzsystemen für manuelle Montagetätigkeiten auf betriebliche Funktionen}

\subsubsection{Betroffene Tätigkeiten}

Der Einsatz und die Verwendung des motionEAP-Montageassistenzsystem betrifft vor allem das direkte Montageumfeld. Hierzu gehören der ausführende Montagemitarbeiter sowie ein montageverantwortlicher Vorarbeiter. Beim Einsatz des motionEAP-Assistenzsystems lassen sich die ausführenden Montagemitarbeiter in die zwei Anwendergruppen der leistungsgeminderten und der normal leistungsfähigen Mitarbeiter unterteilen. Das Assistenzsystem wurde zwar im Speziellen für leistungsgeminderte und leistungsgewandelte Mitarbeiter entwickelt, lässt sich aber durch die intuitive Gestaltung auch für die Nutzergruppe der normal leistungsfähigen Mitarbeiter einsetzen. Die drei hauptsächlich durch das System betroffenen Tätigkeitsfelder zeichnen sich zum bisherigen Zeitpunkt durch folgende Eigenschaften und Arbeitsinhalte aus:

\section{Leistungsgeminderte Mitarbeiter}

Die leistungsgeminderten Mitarbeiter, welche im Zuge des Projektes das Assistenzsystem in zahlreichen Studien evaluiert und getestet haben, lassen sich anhand ihrer Leistungsfähigkeit in die drei Hauptgruppen 0 bis $<15 \%, 15$ bis $35 \%$ und $35 \%+$ einteilen. Diese Unterscheidung erfolgt anhand des Leistungsgrades der Mitarbeiter, welcher sich ausgehend von dem Leistungsgrad eines normal leistungsfähigen Menschen mit $100 \%$ orientiert. Zusätzlich fand für Evaluierungen eine Unterteilung der Behinderungsarten in körperliche, geistige und psychische Beeinträchtigungen statt. Die Ergebnisse verschiedener Studien haben gezeigt, dass vor allem die schwächste Leistungsgruppe der 0 bis $<15 \%$ igen Mitarbeiter mit geistiger Behinderung von einem solchen Montageassistenzsystem profitiert (Bächler et al. 2015; Funk et al. 2015a). Die nachfolgenden Bewertungen erfolgen daher stellvertretend für diese Zielgruppe. 
Aufgrund der begrenzten Einsatz- und Leistungsfähigkeit der Mitarbeiter mit dem Leistungsgrad 0 bis $<15 \%$ umfassen die bisherigen Tätigkeiten vor allem sehr einfache, sich wiederholende und monotone Aufgaben mit geringen Arbeitsinhalten. Hierzu gehören z. B. das Bestücken von Steckbrettern mit Bolzen und Schrauben, das Eintauchen von Bauteilen in Korrosionsschutzöle, das Bekleben von Verpackungen usw. Das Angebot an solch einfachen Tätigkeiten ist in den WfbM jedoch sehr begrenzt, weshalb die Mitarbeiter oftmals aufgrund fehlender Aufträge ohne Tätigkeit und Beschäftigung sind. Die meist geringe kognitive Leistungsfähigkeit dieser Mitarbeiter führt dazu, dass bei der Ausübung von komplexeren Arbeitsinhalten viele Fehler auftreten, die Mitarbeiter eine hohe subjektive Beanspruchung erleben und lange Montagezeiten entstehen (Funk et al. 2015b). Bisher werden die Mitarbeiter durch persönliche Betreuer in ihren Arbeitstätigkeiten eingewiesen und bei Bedarf längerfristig betreut. Zusätzlich bieten am Arbeitsplatz angebrachte Bildanleitungen in Papierform eine weitere Hilfestellung. Die größten Herausforderungen bei leistungsgeminderten Mitarbeitern stellen die große Varianz der Leistungsgrade, die individuellen Beeinträchtigungsarten und die dauerhaft erforderliche technische und pädagogische Betreuung der Mitarbeiter dar.

\section{Normal leistungsfähige Mitarbeiter}

Das Assistenzsystem wurde neben dem Einsatz in einer WfbM auch in der Montageumgebung von einem der größten deutschen Automobilhersteller getestet. Die dortige Nutzergruppe bestand mehrheitlich aus normal leistungsfähigen Mitarbeitern. An den Evaluierungen waren vereinzelt auch leistungsgewandelte Mitarbeiter beteiligt, diese waren jedoch anonym und konnten somit nicht für Auswertungen herangezogen werden. Die Mehrheit der Nutzer hatte einen auf die Tätigkeit bezogenen fachlichen Hintergrund, z. B. als Industriemechaniker, Werkzeugmacher, Schlosser etc. Die derzeitigen Tätigkeiten der Mitarbeiter umfassen die wesentlichen Vorgänge wie Füge- (z. B. Stecken, Schrauben, Fetten, Clipsen) und Handhabungsprozesse (z. B. Aufnehmen, Ablegen) innerhalb der Montage. In den Untersuchungen mit dieser Nutzergruppe wurde die Montage eines PKW-Starters mit 17 Einzelteilen und 30 Montageschritten als exemplarisches Beispiel ausgewählt. Für dieses Produkt besteht eine Variantenvielfalt von über 80 verschiedenen Typen, welche sich oftmals nur geringfügig voneinander unterscheiden. Die hohe Anzahl an Einzelteilen, Montageschritten und Varianten führt unter den derzeitigen Montagebedingungen mit einer anfänglichen Einweisung durch einen Gruppenleiter oder Meister und anschließender eigenverantwortlichen Arbeitsweise mit alleiniger Hilfestellung durch eine Arbeitsanweisung zu einer relativ hohen kognitiven Beanspruchung der Mitarbeiter und einem hohem Fehlerpotenzial.

\section{Gruppenleiter/Meister}

Bis zum heutigen Zeitpunkt erfolgen das Einlernen und die Unterweisung von unerfahrenen Mitarbeitern in neue Montagetätigkeiten in der Regel in vier Stufen. In einer ersten Stufe erfolgt eine mündliche Unterweisung durch den Einweisenden, in einer zweiten Stufe wird der entsprechende Montageprozess vorgemacht, in der dritten Stufe führt der Unterwiesene den Montagevorgang unter Aufsicht des Einweisenden aus und in einer 
letzten Stufe muss der Unterwiesene die Tätigkeit (alleine oder unter Aufsicht) so lange ausführen bzw. einüben, bis dieser die Tätigkeit problemlos beherrscht. In der Industrie erfolgt die Arbeitsunterweisung meist durch einen erfahrenen Vorarbeiter, welcher oftmals die berufliche Qualifikation eines Meisters innehat. In einer WfbM erfolgt die Unterweisung durch einen Gruppenleiter, welcher zum einen Erfahrungen in dem auszuführenden Montageprozess hat, aber auch die persönlichen Fähigkeiten und Besonderheiten des zu Unterweisenden kennt. Aus diesem Grund haben die Gruppenleiter meist sowohl einen technischen Hintergrund und zusätzlich eine Weiterqualifikation im pädagogischen Bereich. Als anschließende Hilfestellung befindet sich sowohl in der Industrie als auch in den WfbMs gewöhnlich eine Arbeitsanweisung in Papierform mit einer Text- und Bildanleitung an den Arbeitsplätzen.

Ein weiterer wichtiger Tätigkeitsschwerpunkt der Gruppenleiter und Meister ist die kurzfristige Planung und Steuerung der einzelnen Montageaufträge sowie die Zuteilung der Montagemitarbeiter auf die jeweiligen Arbeitsplätze.

\subsubsection{Bewertungskriterien}

Die Bewertungskriterien werden detailliert von Wischmann \& Hartmann sowie von Bauer et al. in diesem Band beschrieben.

\subsubsection{Auswirkungen auf betriebliche Funktionen}

Die nachfolgende Tabelle (Tab. 3.1) soll eine Einschätzung der Auswirkungen und Konsequenzen eines Einsatzes des Assistenzsystems zur Unterstützung und Kontrolle von manuellen Montagetätigkeiten auf verschiedene betriebliche Funktionen geben. Die Bewertung der einzelnen Kriterien stellt eine subjektive Einschätzung dar, welche auf ersten Erfahrungen in qualitativen und quantitativen Labor- und Felduntersuchungen des Assistenzsystems mit leistungsgeminderten und normal leistungsfähigen Mitarbeitern basiert.

Tab. 3.1 zeigt, dass sich vor allem für die Nutzergruppe der leistungsgeminderten Mitarbeiter eine positive Veränderung ergibt. Dies äußert sich insbesondere in einer starken Zunahme des Bedarfs und des informellen Lernens. Dieser Anstieg bedeutet, dass bisher nur schwer einsetzbare Mitarbeiter durch die Unterstützung des Systems nun auch für Füge- bzw. Montageprozesse eingesetzt werden können und dadurch ihr Leistungsgrad bzw. die Möglichkeit des informellen Lernens steigt. Bedingt durch diese Veränderungen steigt für diese Mitarbeitergruppe auch der Anteil an komplexen Aufgaben, wobei der Einsatz des Assistenzsystems ein Anstieg der kognitiven Beanspruchung verhindern soll. Eine weitere wichtige Verbesserung ist die Zunahme des Selbstbestimmungsgrads dieser Zielgruppe, denn durch die Verwendung des Assistenzsystems können die Mitarbeiter zwischen verschiedenen Arbeitstätigkeiten auswählen, ihr eigenes Arbeitstempo bestimmen und auch selbstständig die Reihenfolge der auszuführenden Tätigkeiten (insofern prozesstechnisch möglich) festlegen. Der Einsatz der drei Feedback-Level bietet den 
Tab. 3.1 Auswirkungen auf die betrieblichen Funktionen

\begin{tabular}{l|l|l|l}
\hline & $\begin{array}{l}\text { Leistungsgeminderte } \\
\text { Mitarbeiter }\end{array}$ & $\begin{array}{l}\text { Normal leistungsfähige } \\
\text { Mitarbeiter }\end{array}$ & $\begin{array}{l}\text { Gruppenleiter/ } \\
\text { Meister }\end{array}$ \\
\hline Bedarf & ++ & + & -- \\
\hline Problemlösung & -- & - & - \\
\hline Monotone Aufgaben & -- & - & - \\
\hline Komplexe Aufgaben & + & + & - \\
\hline Planen & 0 & 0 & -- \\
\hline Kontrolle & 0 & -- & - \\
\hline Lernen, informell & ++ & + & 0 \\
\hline Lernen, formell & + & + & + \\
\hline Selbstbestimmung & + & + & 0 \\
\hline Optimierung & + & + & + \\
\hline Kooperation & + & + & 0 \\
\hline Kommunikation & 0 & 0 & - \\
\hline Interdisziplinarität & 0 & - & + \\
\hline IT-Kenntnisse & 0 & 0 & + \\
\hline
\end{tabular}

Legende: -- wird viel weniger, - wird weniger, 0 bleibt gleich, + wird mehr, ++ wird viel mehr.

Mitarbeitern die Möglichkeit zur Optimierung der eigenen Tätigkeit. Gleichzeitig wird dadurch ein Anreiz zur Verbesserung und Steigerung der eigenen Effizienz geboten und das Lernen gefördert. Mit entsprechender Erfahrung an einem Einzelarbeitsplatz können selbst schwache Mitarbeiter für die Arbeit an verketteten Arbeitsplätzen befähigt und die Kooperation zwischen Mitarbeitern gefördert werden. Durch die Vernetzung der Assistenzarbeitsplätze können je nach Leistung der verschiedenen Arbeitsplätze einzelne Arbeitsinhalte flexibel auf die anderen Arbeitsplätze verteilt werden. Dadurch können selbst Mitarbeiter mit unterschiedlichen Leistungsniveaus gemeinsam an einer Montagelinie arbeiten. Die einzige Voraussetzung ist eine Variabilität der Montagestruktur und eine Doppelbelegung von entsprechenden Einzelteilen an mehreren Arbeitsplätzen.

Alle diese Aspekte können einen wichtigen Ansatz zur zielgerichteten Unterstützung und Förderung der Inklusion und Partizipation von Menschen mit Einschränkungen in die bzw. an der Arbeitswelt darstellen.

\subsubsection{Zusammenfassende Betrachtung der Auswirkungen}

Abschließend werden die wichtigsten Änderungen eines Arbeitssystems durch den Einsatz des Assistenzsystems betrachtet. Die Erläuterung erfolgt dabei anhand der Systematisierung der Arbeitsbedingungen durch die nachfolgenden Elemente eines Arbeitssystems: 


\section{Arbeitsaufgabe}

Erste Erfahrungen und Ergebnisse zeigen, dass bei leistungsgeminderten Mitarbeitern durch den Einsatz des Assistenzsystems der Inhalt der Arbeitsaufgaben bzw. die Anzahl der Arbeitstätigkeiten pro Arbeitsaufgabe und damit der Leistungsgrad erhöht werden kann. Diese Veränderung geschieht, ohne dabei eine Steigerung der kognitiven Beanspruchung zu bewirken.

\section{Arbeitstätigkeiten}

Im höchsten Feedback-Level des „Experten“ kann die zeitliche Abfolge der Montageaufgaben variabel verändert werden, solange der Prozess bzw. das Produkt dies zulässt. Die räumliche Abfolge, z. B. durch Beidhandarbeit und Mehrfachentnahme von Kleinteilen (z. B. einer Handvoll Schrauben) kann durch eine Abschaltung der Entnahmekontrolle und die alleinige Nutzung der Montagekontrolle flexibel verändert werden.

\section{Arbeitsplatz/Arbeitsraum}

Der geringe zeitliche und inhaltliche Aufwand für das Einrichten eines neuen Montageprozesses ermöglicht eine jederzeitige Veränderung der räumlichen Gestaltung des Arbeitsraumes und der Anordnung der Arbeitsmittel wie z. B. KLT, Schrauber und sonstige Montagewerkzeuge. Der Einrichtevorgang sollte jedoch durch qualifiziertes Personal (Gruppenleiter oder Meister) erfolgen. Auch ein Wechsel der zu montierenden Produkte ist auf diese Weise jederzeit kurzfristig und ohne großen Aufwand möglich.

\section{Arbeitsmittel}

Für einen Einsatz des Assistenzsystems werden hardwareseitig ein PC, ein Projektor und eine Tiefenkamera sowie die Assistenzsoftware benötigt. Um eine digitale Vernetzung eines einzelnen Assistenzarbeitsplatzes mit einer übergeordneten ERP-Software (Enterprise-Ressource-Planning) oder anderen Arbeitsplätzen zu erreichen, wird zusätzlich ein Netzwerk bzw. eine Datenbank benötigt. Es ist allerdings auch die Verwendung eines einzelnen autarken Assistenzsystems möglich.

Des Weiteren können Montagewerkzeuge wie Hämmer und Schraubendreher über fest definierte Ablagepositionen in den Arbeitsprozess eingebunden werden. Zusätzlich können auch Werkzeuge, wie z. B. Handschrauber mit einer Drehmomentüberwachung in das System eingebunden und vernetzt werden.

\section{Arbeitsorganisation}

Der bisher übliche Einlernprozess durch ein zeitaufwändiges Unterweisen, Vormachen und Begleiten kann durch das Assistenzsystem stark reduziert und teilweise sogar ersetzt werden. Denn wenn das Assistenzsystem für ein Produkt einmal eingerichtet ist, steht diese Anleitung jederzeit zur Verfügung. Das System kann autark und ohne menschliche Unterstützung die Unterweisung eines unerfahrenen normal leistungsfähigen Mitarbeiters übernehmen. Bei leistungsgeminderten Mitarbeitern ist jedoch eine zusätzliche Begleitung der Mitarbeiter während der Unterweisung ratsam, um etwaige kognitive Beanspruchungen durch den Neuheitscharakter des Prozesses, des Systems oder des Umfelds zu vermeiden. 
Zusätzlich bietet das System Vorarbeitern eine hohe Flexibilität in ihrem zusätzlichen Aufgabenfeld der Steuerung und Planung von Montageaufträgen. Mithilfe des Systems können die zur Verfügung stehenden Mitarbeiter flexibel auf verschiedenste Arbeitsplätze oder Montageprozess verteilt und zugeordnet werden, ohne dass hierfür der bisherige Erfahrungsgrad berücksichtigt werden muss. Den Gruppenleitern von leistungsgeminderten Mitarbeitern steht durch den Einsatz des Assistenzsystems eine größere Anzahl an leistungsfähigen Mitarbeitern zur Verfügung. Denn gerade auch schwächere Mitarbeiter mit einem Leistungsgrad von 0 bis $<15 \%$, welche bisher nur einfachste Handhabungstätigkeiten ausüben konnten, stehen nun auch für Montage- und Fügeprozesse zur Verfügung.

\section{Arbeitsumgebung}

Der Arbeitsbereich, auf welchen das Feedback projiziert wird, sollte möglichst keiner direkten Sonneneinstrahlung ausgesetzt sein, sondern gleichbleibende Beleuchtungsverhältnisse vorweisen. Mithilfe der intuitiven und sprachfreien Unterweisung durch das Assistenzsystem spielen auch soziale und sprachliche Unterschiede zwischen Montagemitarbeitern und Einweisern zukünftig eine untergeordnete Rolle.

\section{Qualifikation/Kompetenz}

Durch die Unterstützung des Assistenzsystems ist selbst für komplexere Tätigkeiten mit einer hohen Anzahl an Arbeitsschritten nicht mehr länger eine hohe Qualifikation und Fachkompetenz erforderlich. Selbst von unerfahrenen oder leistungsgeminderten Mitarbeitern können bereits beim ersten Montagedurchlauf Aufträge mit hoher Variantenvielfalt ohne vorherige Einweisung fehlerfrei und mit niedriger kognitiver Beanspruchung durchgeführt werden.

\subsection{Danksagung}

Dieser Beitrag ist im Rahmen des Projekts „,motionEAP - System zur Effizienzsteigerung und Assistenz bei Produktionsprozessen in Unternehmen auf Basis von Bewegungserkennung und Projektion“ entstanden. Das Projekt wurde vom Bundesministerium für Wirtschaft und Energie (BMWi) unter dem Kennzeichen 01MT12021D gefördert und vom DLR-Projektträger betreut.

\section{Literatur}

Adenauer, S. (2004). Die (Re-) Integration leistungsgewandelter Mitarbeiter in den Arbeitsprozess. Das Projekt FILM bei FORD Köln. angew. Arbeitswiss, 181, 1-18.

Arnold, D., \& Furmans, K. (2009). Materialfluss in Logistiksystemen. Berlin: Springer.

Artikel des Sozialgesetzbuches - Neuntes Buch - (SGBIX) Rehabilitation und Teilhabe behinderter Menschen. (2001). Sozialgesetzbuch (SGB) Neuntes Buch (IX) - Rehabilitation und Teilhabe behinderter Menschen - (SGB IX). 
Bächler, L., Bächler, A., Kölz, M. et al (2015). Über die Entwicklung eines prozedural-interaktiven Assistenzsystems für leistungsgeminderte und -gewandelte Mitarbeiter in der manuellen Montage. In Soeffke Uni Magdeburg (Hrsg.), Tagung Mensch Maschine. Magdeburg.

Beauftragte der Bundesregierung für die Belange behinderter Menschen. (2014). Die UN-Behindertenrechtskonvention. Übereinkommen über die Rechte von Menschen mit Behinderungen. Berlin: Beauftragte der Bundesregierung für die Belange behinderter Menschen.

Böhle, F. (2005). Erfahrungswissen - die verborgenen Seiten professionellen Handelns. Eine Herausforderung für die berufliche Bildung. Bonn: Fachtagung des Bundesinstituts für Berufsbildung.

Bundesministerium für Arbeit und Soziales. (2011). Übereinkommen der Vereinten Nationen über Rechte von Menschen mit Behinderungen. Vom Bundeskabinett beschlossen am 3. August 2011. Bonn.

Bundesministerium für Arbeit und Soziales. (2014). Initiative Inklusion. Maßnahmen zur Förderung der Teilhabe schwerbehinderter Menschen am Arbeitsleben auf dem allgemeinen Arbeitsmarkt.

Bundesministerium für Wirtschaft und Technologie (BMWi). (2013). Engpassanalyse 2013. Besondere Betroffenheit in den Berufsfeldern Energie und Elektro sowie Maschinen- und Fahrzeugtechnik. Berlin.

Funk, M., Baechler, A., Baechler, L. et al. (2015a). Comparing projected in-situ feedback at the manual assembly workplace with impaired workers. In Proceedings of the 8th International Conference on Pervasive Technologies Related to Assistive Environments, New York, NY, USA.

Funk, M., Mayer, S., \& Schmidt, A. (2015b). Using in-situ projection to support cognitively impaired workers at the workplace. In Proceedings of the 17th International ACM SIGACCESS Conference on Computers \& Accessibility.

Günthner, W. A., Schedlbauer, M., \& Wulz, J. (2004). Augmented Reality in der innerbetrieblichen Logistik. wt Werkstattstechnik-online, 07/08, 363-365.

Hörz, T., Korn, O., \& Kölz, M. (2013). Assistenzsysteme für leistungseingeschränkte Mitarbeiter in der manuellen Montage. In Abschlussbericht Innovative Projekte/Kooperationsprojekte. Baden-Württemberg, Germany: Koordinierungsstelle Forschung der Hochschulen für Angewandte Wissenschaften.

Jahn, H.-P. (2001). Datenerfassung und -verarbeitung bei der ergonomischen Gestaltung von Arbeitsplätzen-mehrere Jahre nach Abschluss eines HdA-Projektes für Leistungsgewandelte. Herbstkonferenz GfA.

Kant, I. (1961). Grundlegung zur Metaphysik der Sitten (429 S). Stuttgart: Reclam Verlag.

Lotter, B. (2012). Überlegungen zum Montagestandort Deutschland. In Montage in der industriellen Produktion (S. 1-8). Berlin: Springer.

Reif, R. (2009). Entwicklung und Evaluierung eines Augmented Reality unterstützten Kommissioniersystems. München: FML.

Reinhart, G., \& Zäh, M. (2014). Assistenzsysteme in der Produktion. wt Werkstattstechnik-online, 104(9), 516.

Sauer, D. (2011). Von der „Humanisierung der Arbeit“ zur „Guten Arbeit“. APuZ, 15/2011, 18-24.

Schaper, N. (2006). Bedeutung von Arbeit und Arbeitsmotivation. Vorlesung Arbeits- und Personalpsychologie. Lehrstuhl für Arbeits- und Organisationspsychologie. Paderborn: Universität Paderborn.

Schuntermann, M. F. (2007). Einführung in die ICF. Grundkurs, Übungen, offene Fragen. Landsberg/Lech: Ecomed Medizin.

Schwab, H. (2009). Jugendhilfe und Integration in Arbeit. In M. Lindenau (Hrsg.), Jugend im Diskurs - Beiträge aus Theorie und Praxis. Festschrift zum 60. Geburtstag von Jürgen Gries (S. 119-130). Wiesbaden: Springer Fachmedien. 
Semmer, N., \& Udris, I. (2004). Bedeutung und Wirkung von Arbeit. In H. Schuler (Hrsg.), Lehrbuch Organisationspsychologie (S. 157-195). Bern: Huber.

Statistisches Bundesamt. (2009). Bevölkerung Deutschlands bis 2060. 12. koordinierte Bevölkerungsvorausberechnung. Wiesbaden.

Ten Hompel, M., Sadowsky, V., \& Beck, M. (2011). Kommissionierung, Materialflusssysteme 2 Planung und Berechnung der Kommissionierung in der Logistik. Berlin: Springer.

Open Access Dieses Kapitel wird unter der Creative Commons Namensnennung 4.0 International Lizenz (http://creativecommons.org/licenses/by/4.0/deed.de) veröffentlicht, welche die Nutzung, Vervielfältigung, Bearbeitung, Verbreitung und Wiedergabe in jeglichem Medium und Format erlaubt, sofern Sie den/die ursprünglichen Autor(en) und die Quelle ordnungsge-mäß nennen, einen Link zur Creative Commons Lizenz beifügen und angeben, ob Änderungen vorgenommen wurden.

Die in diesem Kapitel enthaltenen Bilder und sonstiges Drittmaterial unterliegen ebenfalls der genannten Creative Commons Lizenz, sofern sich aus der Abbildungslegende nichts anderes ergibt. Sofern das betreffende Material nicht unter der genannten Creative Commons Lizenz steht und die betreffende Handlung nicht nach gesetzlichen Vorschriften erlaubt ist, ist für die oben aufgeführten Weiterverwendungen des Materials die Einwilligung des jeweiligen Recht-einhabers einzuholen.

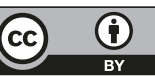

